\title{
TOLERANCIA IN VITRO DE FUSARIUM SOLANI Y FUSARIUM SEMITECTUM (ANAMORFOS, AsCOMYCOTA) A LA EXPOSICIÓN A DESINFECTANTES DE SUPERFICIE UTILIZADOS EN ACUICULTURA
}

\author{
SUANI G. PACHECO MARINO ${ }^{1,2}$, MARTA N. CABELLO ${ }^{1,3}$ y ALFREDO SALIBIÁN ${ }^{3,4}$
}

\begin{abstract}
Summary: In vitro tolerance of Fusarium semitectum and Fusarium solani (anamorphic, Ascomycota) exposed to surface disinfectant commonly used in aquaculture. An in vitro evaluation of the antifungal effect of acetic acid $(\mathrm{AcH})$, buffered acetic acid $(\mathrm{AcH}+\mathrm{AcNa})$, potassium iodide $(\mathrm{KI})$ and lodopovidone (IP); on the growth rate (GR) of Fusarium solani (Mart.) Sacc. 1881 and Fusarium semitectum Berk. \& Ravenel, 1875 was performed. Both fungal species were isolated from eggs of the Argentinian silverside Odontesthes bonariensis (Cuvier \& Valenciennes, 1835). The studied substances are commonly used in fish farming as surface disinfectants. The tested concentrations of this substances exhibited antifungal activity for both strains at concentrations higher than their 96h median lethal concentrations (LC50-96h) for the treated eggs; concentrations below the (LC50-96h) exhibited little or no effect.
\end{abstract}

Key words: Acetic acid; fungicides, in-vitro growth inhibition, lodophors, Odontesthes bonariensis eggs.

\begin{abstract}
Resumen: Se evaluó in vitro el efecto del: acido acético $(\mathrm{AcH})$, ácido acético tamponado $(\mathrm{AcH}+\mathrm{AcNa})$, ioduro de potasio (IK) y lodopovidona (IP) sobre la tasa de crecimiento (TC) de Fusarium solani (Mart.) Sacc. 1881 y Fusarium semitectum Berk. \& Ravenel, 1875. Ambas especies fueron aisladas de ovas de Odontesthes bonariensis (Cuvier \& Valenciennes, 1835). Las sustancias estudiadas son comúnmente utilizadas en acuicultura como desinfectantes de superficie. Las concentraciones ensayadas con actividad fungicida para ambas cepas fueron mucho más elevadas que las correspondientes concentraciones letales medias a 96 horas (CL50-96h) para ovas de pejerrey. Concentraciones inferiores a la CL50 - 96h exhibieron un efecto fungistático escaso o nulo.
\end{abstract}

Palabras clave: Ácido acético; fungicidas; inhibición del crecimiento in-vitro; lodóforos; ovas de Odontesthes bonariensis.

\section{INTRODUCCIÓN}

Las infecciones producidas por especies fúngicas del género Fusarium han sido descritas en gran variedad de organismos acuáticos, tanto de vida libre como en cautiverio (Hatai et al., 1986; Crow et

\footnotetext{
${ }^{1}$ Instituto de Botánica C. Spegazzini, Facultad de Ciencias Naturales y Museo, Universidad Nacional de La Plata, Calle 53 № 477, B1900AVJ - La Plata, Buenos Aires, Argentina. (suani.pm@gmail.com).

2 Consejo Nacional de Investigaciones Científicas y Técnicas (CONICET), Av. Rivadavia 1917, C1033AAJ Buenos Aires, Argentina.

${ }^{3}$ Comisión de Investigaciones Científicas $(\mathrm{CIC})$ de la Pcia. de Buenos Aires, Calle 526, entre 10 y 11, 1900 - La Plata, Argentina.

${ }^{4}$ Departamento de Ciencias Básicas (PRODEA-INEDES), Universidad Nacional de Luján, Casilla de Correo 221, B6700ZBA - Luján, Argentina.
}

al., 1995; Souheil et al., 1999; Khoa et al., 2004). Si bien, la magnitud de las repercusiones económicas de dichas infecciones son aún desconocidas, el efecto de la Fusariosis cobra importancia económica en el cultivo intensivo de crustáceos debido a las elevadas mortalidades que provoca.

La evaluación de potenciales agentes fungicidas destinados al tratamiento de infecciones causadas por organismos del Reino Fungi, es un temática que ha sido poco estudiada. Bell et al. (1990) evaluaron la eficacia in vivo del BG 101 (2-metil-4isotiazolina-3-ona y 5-Cloro-2-metil-4-isotiazolina3-ona) en el control de mortalidades causadas por F. solani (Mart.) Sacc. 1881, en el camarón azul Penaeus stylirostris (Stimpson, 1874), pero su uso como fungicida está restringido al tratamiento de crustáceos debido a que dicha sustancia es muy tóxica para los peces. En la actualidad, las 
medidas de control de esta enfermedad en el cultivo intensivo de crustáceos se limitan a la profilaxis y prevención (Victoria Alday-Sanz com. pers.).

Por su parte, en el cultivo de peces, los tratamientos fungicidas se enfocan principalmente en control y prevención de la Saprolegniasis, habiéndose encontrado escasa información sobre el efecto que dichos fungicidas ejercen en organismos del Reino Fungi. En estudios previos realizados en nuestro laboratorio, se determinó y comparó la acción fungicida del cloruro de sodio, verde de malaquita y la formalina, encontrando que $F$. solani (Mart.) Sacc. 1881 y F. semitectum Berk. \& Ravenel 1875 toleran elevadas concentraciones de dichas sustancias (Pacheco Marino et al., 2011).

Este trabajo tiene por objetivo la evaluación in vitro del potencial fungicida del ácido acético y de dos iodóforos, sustancias empleadas en acuicultura como desinfectantes de superficie, utilizando como especies blanco a F. solani y F. semitectum.

\section{Materiales y Métodos}

Se evaluó el efecto fungicida del: ácido acético (glacial) $(\mathrm{AcH})$, ácido acético tamponado $(\mathrm{AcH}+\mathrm{AcNa})$, ioduro de potasio (IK) y Iodopovidona (IP). Se utilizaron reactivos de grado analítico; la Iodopovidona fue un formulado comercial al $10 \%$ ("Pervinox"). Para la selección del rango de concentraciones a ensayar se consideraron las concentraciones letales medias a 96 horas (CL5096h) y las Concentraciones de No Efecto (NEC) de los fungicidas para ovas de pejerrey en el estadio "ojos pigmentados" 147 horas post fecundación (Chalde et al., 2011), las que fueron determinadas en nuestro laboratorio (Pacheco Marino \& Salibián, 2010). Los bioensayos de efecto fungicida se realizaron con Fusarium semitectum (LPS 1001) y F. solani (LPS 1002), ambas cepas fueron aisladas de ovas de pejerrey Odontesthes bonariensis y depositadas en la colección de cultivos del Instituto de Botánica C. Spegazzini.

Se utilizó como medio base Agar Czapek-Dox modificado (Kirk et al., 2001), con agregado de cada sustancia en estado puro, exceptuando al IK que fue añadido en una solución al $1 \%$, hasta obtener las siguientes concentraciones nominales mencionadas $\mathrm{mg} . \mathrm{L}^{-1}$ :

- AcH: 0 (control), 10-15-25-50-100-200-500-1000.
- AcH+AcNa: 0 (control), 100-200-400-600-8001000-1500-2000.

- IK: 0 (control), 20-30-50-72,5-145-240-480-960.

- IP: 0 (control), 30-50-80-120-180-360-720-1440.

Las placas fueron sembradas por cuadriplicado con inóculos de $3 \mathrm{~mm}$ diámetro, provenientes de cultivos monospóricos. Se incubaron a $17{ }^{\circ} \mathrm{C}$, a partir del tercer día de incubación y durante dos semanas se midió el diámetro de las colonias diariamente en la misma dirección, utilizando un calibre Vernier (precisión $=0,1 \mathrm{~mm}$ ), hasta que el grupo Control de alguna de las réplicas alcanzara el borde de la placa. En las placas en las que no se observó crecimiento durante las dos semanas, el ensayo se prolongó por una semana adicional.

Las tasas de crecimiento (TC) para cada concentración fueron expresadas como la pendiente de la línea de regresión y se calculó el coeficiente de correlación de Pearson. La inhibición del crecimiento (IC) se indica como porcentaje de reducción del diámetro de las colonias en los medios con cada sustancia comparándolo con el del grupo control. Este parámetro fue calculado aplicando la siguiente fórmula:

IC $(\%)=100-[100$ (Diámetro de la colonia tratada /Diámetro de la colonia Control)]

Se determinó la existencia de diferencias estadísticamente significativas entre las TC utilizando un ANOVA de una vía y se realizaron comparaciones entre grupos empleando el test de multicomparaciones a posteriori de Dunnet (Zar, 2010), en ambos casos con la ayuda del software XLStat version 7.5.

\section{Resultados}

El crecimiento de ambas especies respondió a un modelo de regresión lineal simple de origen en cero, tal como lo demuestran los valores del Coeficiente de correlación de Pearson. En la Tabla 1 se presentan: la pendiente de la recta de regresión, el coeficiente de correlación de Pearson y los porcentajes de inhibición del crecimiento (IC) para cada especie y sustancia.

Los valores de las pendientes de los controles indican que la TC de F. semitectum fue el doble que la de F. solani (relación 2.0-2.2). Los coeficientes de correlación de Pearson oscilaron en torno a 0,99 (excepto en las concentraciones más elevadas de $\mathrm{AcH}+\mathrm{AcNa})$. 


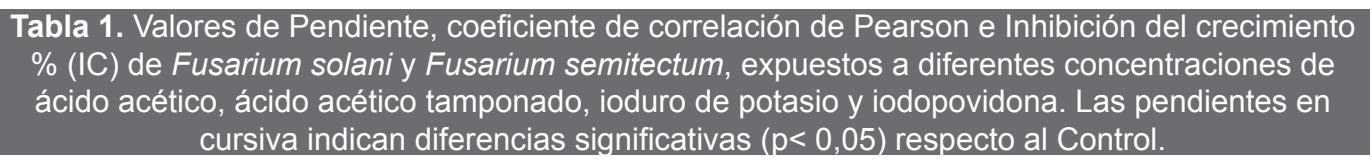

\begin{tabular}{|c|c|c|c|c|c|c|c|}
\hline & \multirow[b]{2}{*}{$\begin{array}{l}\text { Concentración } \\
\text { [mg.L-1] }\end{array}$} & \multicolumn{3}{|c|}{ Fusarium solani } & \multicolumn{3}{|c|}{ Fusarium semitectum } \\
\hline & & Pendiente & $\begin{array}{l}\text { Coeficiente } \\
\text { de Pearson }\end{array}$ & $\begin{array}{l}\text { Inhibición del } \\
\text { crecimiento } \\
(\%)\end{array}$ & Pendiente & $\begin{array}{l}\text { Coeficiente } \\
\text { de Pearson }\end{array}$ & $\begin{array}{c}\text { Inhibición del } \\
\text { crecimiento } \\
(\%)\end{array}$ \\
\hline \multirow{10}{*}{ 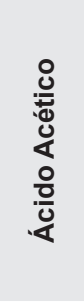 } & 0 & 0,476 & 1.000 & & 1.007 & 0,996 & \\
\hline & 10 & 0,484 & 0,998 & 0,13 & 1.000 & 0,999 & $-0,80$ \\
\hline & 15 & 0,476 & 1.000 & 2,67 & 0,979 & 0,999 & 1,06 \\
\hline & 25 & 0,479 & 0,999 & 0,13 & 1.002 & 0,999 & $-1,11$ \\
\hline & 50 & 0,464 & 0,999 & 3,94 & 0,979 & 0,999 & 0,00 \\
\hline & 100 & 0,489 & 1.000 & $-0,51$ & 0,973 & 0,999 & $-0,16$ \\
\hline & 200 & 0,508 & 1.000 & $-4,96$ & 0,987 & 0,999 & 0,00 \\
\hline & 500 & 0,397 & 0,993 & 12,47 & 0,706 & 0,999 & 22,17 \\
\hline & 1000 & 0,014 & 0,577 & 91,09 & 0,063 & 0,839 & 86,62 \\
\hline & 2000 & & & 100,00 & & & 100,00 \\
\hline \multirow{9}{*}{ 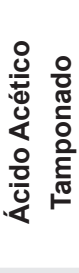 } & 0 & 0,370 & 0,993 & & 0,808 & 1.000 & \\
\hline & 100 & 0,478 & 1.000 & $-25,00$ & 0,933 & 0,999 & $-11,58$ \\
\hline & 200 & 0,439 & 0,993 & $-19,09$ & 0,881 & 0,999 & $-6,07$ \\
\hline & 400 & 0,354 & 0,984 & $-2,92$ & 0,671 & 0,992 & 11,21 \\
\hline & 600 & 0,236 & 0,952 & 23,90 & 0,488 & 0,983 & 30,33 \\
\hline & 800 & 0,055 & 0,788 & 73,97 & 0,262 & 0,931 & 56,99 \\
\hline & 1000 & & & 100,00 & 0,005 & 0,577 & 98,16 \\
\hline & 1500 & & & 100,00 & & & 100,00 \\
\hline & 2000 & & & 100,00 & & & 100,00 \\
\hline \multirow{9}{*}{ 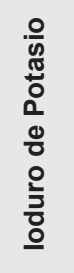 } & 0 & 0,315 & 0,998 & & 0,642 & 0,997 & \\
\hline & 20 & 0,312 & 0,988 & $-5,77$ & 0,636 & 0,997 & 3,49 \\
\hline & 30 & 0,286 & 0,990 & 0,96 & 0,613 & 0,999 & 6,11 \\
\hline & 50 & 0,306 & 0,992 & $-4,81$ & 0,630 & 0,998 & 3,06 \\
\hline & 72,5 & 0,302 & 0,992 & $-6,73$ & 0,629 & 0,996 & 1,75 \\
\hline & 145 & 0,313 & 0,991 & $-5,77$ & 0,605 & 0,995 & 4,80 \\
\hline & 240 & 0,262 & 0,992 & 7,69 & 0,553 & 0,992 & 10,92 \\
\hline & 480 & 0,268 & 0,991 & 7,69 & 0,530 & 0,990 & 11,79 \\
\hline & 960 & 0,245 & 0,982 & 10,58 & 0,400 & 0,979 & 28,82 \\
\hline \multirow{9}{*}{ 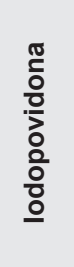 } & 0 & 0,308 & 0,986 & & 0,642 & 0,997 & \\
\hline & 30 & 0,317 & 0,990 & $-2,88$ & 0,642 & 0,996 & $-0,44$ \\
\hline & 50 & 0,296 & 0,992 & $-0,96$ & 0,642 & 0,996 & $-1,31$ \\
\hline & 80 & 0,322 & 0,988 & $-5,77$ & 0,643 & 0,994 & $-2,18$ \\
\hline & 120 & 0,306 & 0,992 & $-5,77$ & 0,643 & 0,997 & $-0,44$ \\
\hline & 180 & 0,312 & 0,990 & $-4,81$ & 0,610 & 0,993 & 2,18 \\
\hline & 360 & 0,289 & 0,990 & 0,00 & 0,491 & 0,985 & 14,85 \\
\hline & 720 & 0,253 & 0,973 & 3,85 & 0,177 & 0,870 & 57,64 \\
\hline & 1440 & 0,271 & 0,974 & 0,00 & & & 100,00 \\
\hline
\end{tabular}

En la Fig. 1 A-B se ilustra el efecto del AcH y el $\mathrm{AcH}+\mathrm{AcNa}$ sobre el crecimiento de ambas especies fúngicas al octavo día de exposición. Podemos observar que el $\mathrm{AcH}$ no inhibe el crecimiento de ambas especies a concentraciones inferiores a la CL-50 96h para ovas de pejerrey (228 mg. $\left.\mathrm{L}^{-1}\right)$. Sin embargo, se encontraron diferencias estadísticamente significativas entre las TC de los Controles y las placas expuestas a 500 y $1000 \mathrm{mg} \cdot \mathrm{L}^{-1}$, en donde la IC fue de 12,47 y $91,09 \%$ para $F$. solani y 22,17 y $86,62 \%$ para $F$. semitectum. A diferencia del caso anterior, el AcH $+\mathrm{AcNa}$ (Fig. 1B) exhibió un efecto levemente fungistático a concentraciones inferiores a la CL-50 96h (704 mg.L $\left.\mathrm{L}^{-1}\right)$. Se encontraron diferencias estadísticamente significativas entre las TC del
Control y las placas de $F$. semitectum expuestas a concentraciones mayor iguales a $400 \mathrm{mg} \cdot \mathrm{L}^{-1}(\mathrm{p}<0,05)$, donde la IC fue $11,21 \%$. Para $F$. solani se encontraron diferencias estadísticamente significativas $(p<0,05)$ en las TC del Control y las placas expuestas a concentraciones mayores iguales a $600 \mathrm{mg} \cdot \mathrm{L}^{-1}$. En ambos casos, esta sustancia actuó como fungicida a concentraciones superiores a $800 \mathrm{mg} \cdot \mathrm{L}^{-1}$.

En la Fig. 1 C-D se grafican los resultados referidos al efecto inhibitorio de los dos compuestos iodados ensayados, ninguna de las concentraciones tuvieron efecto fungicida. El efecto fungistático del IK (Fig. 1C) fue observado en concentraciones superiores a la CL50 96h para ovas de pejerrey (147 mg.. $\left.\mathrm{L}^{-1}\right)$. En ambas especies, se encontraron diferencias estadísticamente 
significativas en las TC de los controles y las placas expuestas a concentraciones mayor iguales a $240 \mathrm{mg} . \mathrm{L}^{-1}$ de IK. En las placas expuestas a 960 mg. $\mathrm{L}^{-1}$ las IC resultaron en 28,82 y $10,57 \%$ y para $F$. semitectum y $F$. solani, respectivamente. Por su parte, la Iodopovidona tampoco tuvo un efecto inhibidor a concentraciones inferiores a la CL-50 96h para ovas de pejerrey (198 mg. $\left.\mathrm{L}^{-1}\right)$. El crecimiento de $F$. solani no fue inhibido por IP a ninguna concentración; sin embargo, se encontraron diferencias estadísticamente significativas en las TC de F. semitectum expuesto a concentraciones mayor iguales a $360 \mathrm{mg} . \mathrm{L}^{-1}$ (Fig. 1D).

\section{Discusión}

Se observó que tanto para $F$. solani como para F. semitectum el Ácido Acético tuvo un efecto inhibidor a concentraciones superiores a 500 mg. $\mathrm{L}^{-1}$; concentraciones inferiores mostraron un efecto levemente estimulador del crecimiento en $F$. solani y levemente inhibidor en $F$. semitectum. No es de extrañar la capacidad de ambas especies para crecer en las concentraciones de Ácido Acético ensayadas, puesto que la mayoría de hongos del suelo se desarrollan perfectamente en ambientes ligeramente ácidos. Esta cualidad puede verse exacerbada, sobre todo en condiciones de cultivo, donde los organismos disponen de los nutrientes necesarios para poner en marcha los mecanismos de control del medio interno y así lograr contrarrestar el descenso del pH. (Domsh et al., 1993). Bennett et al. (1976) consideran que el Ácido Acético tendría un efecto fungistático, ya que en presencia de glucosa su efecto tóxico puede ser contrarrestado. No obstante, la exposición a concentraciones muy elevadas de $\mathrm{AcH}$ puede ser letal. Kang et al. (2003) encontraron que a una concentración de ácido acético de $3 \mathrm{mg} . \mathrm{L}^{-1}$ el crecimiento de Colletotrichum spp. es totalmente inhibido. Efectos similares fueron observados por Conková et al. (1993) en Penicllium glabrum, Aspergillus flavus, A. funimatus, A. niger, Fusarium moniliforme y Cladosporium sphaerospermum; donde el crecimiento de estas especies fue inhibido al ser expuestas a $50 \mathrm{mg} \cdot \mathrm{L}^{-1}$ de ácido acético.

A
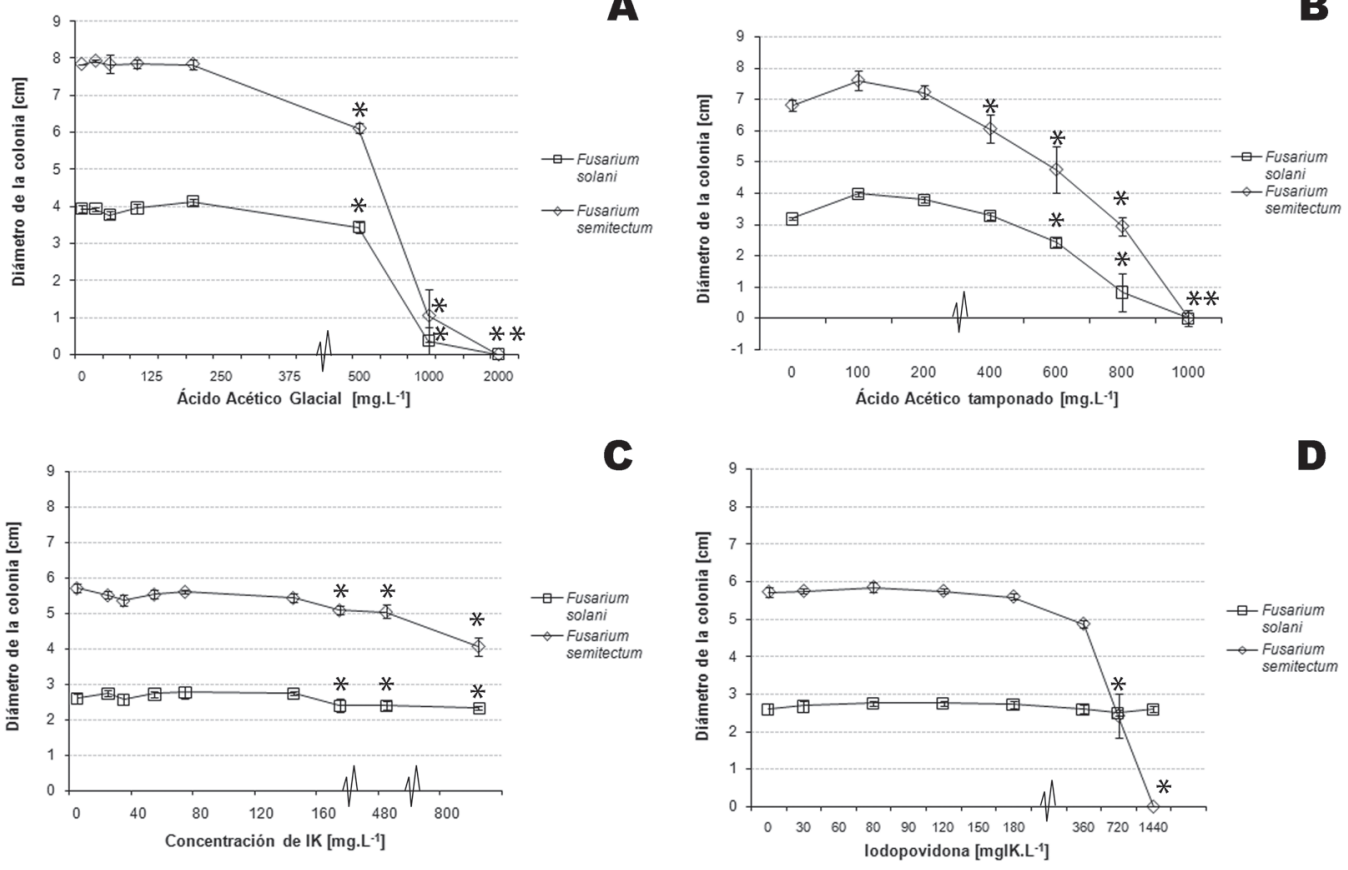

Fig. 1. Crecimiento de Fusarium solani y Fusarium semitectum en Czapeck Dox luego de 8 días de exposición. A: Efecto del Ácido Acético Glacial. B: Ácido Acético tamponado. C: Loduro de Potasio. D: lodopovidona. Los datos son expresados como medias \pm desviación estándar (D.E.) del diámetro de las colonias. *: Diferencias significativas $(p<0,05)$ respecto del Control. 


\section{S. G. Pacheco Marino et al. - Dos especies de Fusarium tolerantes a desinfectantes}

Nosotros registramos una respuesta diferente al exponer a $F$. solani y $F$. semitectum al ácido acético tamponado, donde se observó un efecto inhibidor en 400 y $600 \mathrm{mg} . \mathrm{L}^{-1}$ para $F$. semitectum y F. solani respectivamente, sugiriendo que $F$. semitectum podría ser más sensible al ácido acético que $F$. solani. En la actualidad se desconoce la naturaleza de la toxicidad del acetato, sólo se sabe que su efecto sería más fungistático que fungicida (Shimokawa \& Nakayama, 1999). Munir \& Shimada (2005) estudiaron el efecto del acetato sobre el crecimiento de Basidiomycetes causantes de la pudrición de la madera, y encontraron que una concentración de $10000 \mathrm{mg} . \mathrm{L}^{-1}$ no inhibe el crecimiento de Aspergilllus niger y Chaetomium globossum, pero sí el de otras especies. Bennett et al. (1976) encontraron que Aspergillus parasiticus puede utilizar acetato de sodio y ácido acético como fuente de carbono en ausencia de glucosa para sustentar su crecimiento vegetativo, pero sólo es capaz de emplear el acetato de sodio, aunque de manera menos eficiente, para la síntesis de metabolitos secundarios como toxinas y pigmentos; estos hallazgos llevaron a dichos autores a concluir que el ácido acético tendría solamente un efecto fungistático.

El yodo es considerado, principalmente, un efectivo agente contra bacterias y virus (Wright \& Snow, 1975; Alderman, 1984), y como un efectivo tratamiento para la Saprolegniasis (Subasinghe \& Sommerville, 1985; Schreck et al., 1990, 1992). Sin embargo, existen escasos estudios que hayan abordado el análisis del efecto de los iodóforos sobre organismos del Reino Fungi. Hiruma \& Kagawa (1987) estudiaron el efecto del yodo y el ioduro de potasio sobre el Ascomycota levaduriforme Sporothrix schenckii y encontraron que estas sustancias provocaron alteraciones ultraestructurales y también una disminución en su tasa de germinación. Hallazgos similares fueron reportados por Torres-Mendoza et al. (1997), quienes afirman que tanto el yodo, como ioduro, a una concentración de $39 \mu \mathrm{M}$, son capaces de generar daño celular en hongos levaduriformes. Khodabandeh \& Abtahi (2006), realizaron ensayos en ovas de carpa común infectadas con Saprolegnia sp. y encontraron que el yodo en concentraciones de 50,100 y $200 \mathrm{mg} . \mathrm{L}^{-1}$, tiene un efecto ligeramente fungicida, no siendo efectivo en la inhibición del crecimiento de Saprolegnia sp. En nuestro caso, el ioduro de potasio no ejerció un efecto inhibidor importante sobre las cepas de Fusarium estudiadas en este trabajo, lo que concuerda con lo observado por Khodabandeh \& Abtahi (2006). El crecimiento de $F$. semitectum fue parcialmente inhibido (28,82\%) a una concentración de $960 \mathrm{mg} . \mathrm{L}^{-1}$, una concentración muy elevada para ser utilizada como tratamiento en ovas de pejerrey, ya que se encuentra por encima de su respectiva CL50 - 96h para ovas de pejerrey. Al exponer a ambas especies a la Iodopovidona se observó que las concentraciones ensayadas no tuvieron efecto sobre el crecimiento de $F$. solani, pero inhibieron el crecimiento de $F$. semitectum, habiéndose observado una IC de 56,64 $\%$ en las placas expuestas a $720 \mathrm{mg} . \mathrm{L}^{-1}$ y un efecto letal a $1440 \mathrm{mg} . \mathrm{L}^{-1}$. Esta sensibilidad diferencial no fue observada en los ensayos con IK, lo que sugiere que el efecto inhibitorio de la Iodopovidona sobre $F$. semitectum puede ser atribuido a algún componente del formulado y no al ingrediente activo.

\section{Bibliografía}

ALDERMAN, D. J. 1984. The toxicity of iodophors to salmonid eggs. Aquaculture 40: 7-16.

BELL, T. A., D. V. LIGHTNER \& R. R. WILLIAMS. 1990. A promising new chemotherapeutant for use in treatment of Fusarium solani infections in penaeid shrimp. J. World Aquacult. Soc. 21:131-136.

BENNETT, J. W., L. S. LEE \& G. G. GAAR. 1976. Effect of Acetone on production of Aflatoxins and Versicolorin pigments by resting cell cultures of Aspergillus parasiticus. Mycophatologia 58: 9-12.

CHALDE, T., D. A. FERNÁNDEZ, V. E. CUSSAC \& G. M. SOMOZA. 2011. The effect of rearing temperature in larval development of pejerrey, Odontesthes bonariensis - Morphological indicators of development. Neotrop. Ichthyol. 9: 747-756.

CONKOVÁ, E., L. PARA \& A. KOCISOVA. 1993. Inhibition of the molds growth by selected organicacids. Vet. Med. 38: 723-727.

CROW, G. L., J. A. BROCK \& S. KAISER. 1995. Fusarium solani fungal infection of the lateral line canal system in captive scalloped hammerhead sharks (Sphyrna lewini) in Hawaii. J. Wildlife Dis. 31: 562-565.

DOMSCH, K. H., W. GAMS \& T. A. ANDERSON. 1993. Compendium of soil fungi. Vol. 1. IHWVerlag, Eching.

HATAI, K., S. KUBOTA, N. KIDA \& S. UDAGAWA. 1986. Fusarium oxysporum in Red Sea Bream (Pagrus sp.). J. Wildlife Dis. 22: 570-571. 
HIRUMA, M. \& S. KAGAWA 1987. Ultrastructure of Sporothrix schenckii treated with iodine potassium iodide solution. Mycopathologia 97: 121-127.

KIRK, P. M., P. F. CANNON, J. C. DAVID \& J. A. STALPERS (Eds). 2001. Ainsworth and Bisby's Dictionary of the Fungi. 9th. ed. CABI, Wallingford.

KANG, H. C., Y. H. PARK \& S. J. GO. 2003. Growth inhibition of a phytopathogenic fungus, Colletotrichum species by acetic acid. Microbiol. Res. 158: 321-326.

KHOA, L.V., K. HATAI \& T. AOKI. 2004. Fusarium incarnatum isolated fron black tiger shrimp, Penaeus monodon Fabricius, with black gill diseases culture in Vietnam. J. Fish Dis. 27: 507-515.

KHODABANDEH, S. \& B. ABTAHI. 2006. Effects of sodium chloride, formalin and iodine on the hatching success of common carp, Cyprinus carpio, eggs. J. Appl. Ichthyol. 22: 54-56.

MUNIR, E. \& M. SHIMADA. 2005. An inhibitory effect of acetate added to the culture on growth of Word rotting Basidiomycetes. Proceedings of the 6th International Wood Science Symposium LIPIJSPS Core University Program in the Field of Wood Science, pp. 405-410. Bali.

PACHECO MARINO, S. G. \& A. SALIBIAN. 2010. Acute toxicity of three antifungal chemicals on silverside Odontesthes bonariensis (Valenciennes 1835) eggs. Int. J. Environ. Health 4: 333-341.

PACHECO MARINO, S. G., N. M. CABELLO \& A. SALIBIAN. 2011. Effect of three fungicides on in vitro growth of Fusarium solani and Fusarium semitectum, parasitic on Odontesthes bonariensis eggs. Int. J. Environ. Health 5: 293-303.

SCHRECK, C. B., M. S. FITZPATRICK, L. L. MARKING, J. J. RACH, \& S.,M. JEFFREY. 1990. Research to identify effective antifungal agents. Annual Report, to Bonneville Power Administration, US Department of Energy, Portland, Oregon.

SCHRECK, C. B., M. S. FITZPATRICK, L. L.
MARKING, J. J. RACH \& T. M. SCHREIER. 1992. Research to identify effective antifungal agents. Annual Report, Bonneville Power Administration, US Department of Energy, Portland, Oregon.

SHIMOKAWA, O. \& H. NAKAYAMA. 1999. Acetatemediated growth inhibition in esterol $14-\alpha$ demethylation-deficient cells of Candida albicans. Antimicrob. Agents Chemother. 43:100-105.

SOUHEIL, H., A. VEY, P. THUET \& J. P. TRILLES. 1999. Pathogenic and toxic effects of Fusarium oxysporum (Schlecht.) on survival and osmoregulatory capacity of Penaeus japonicus (Bate). Aquaculture 178: 209224.

SUBASINGHE, R. P. \& C. SOMMERVILLE. 1985. Disinfection of Oreochromis mossambicus (Peters) eggs against commonly occurring potentially pathogenic bacteria and fungi under artificial hatchery conditions. Aquacult. Fish. Manag. 16: 121-127.

TORRES-MENDOZA, B. M., E. VÁZQUEZ-VALLS \& A. GONZÁLEZ-MENDOZA. 1997. Efecto del yoduro de potasio sobre la respuesta inmune en la esporotricosis. Revista Iber. Micol. 14: 98-100.

WRIGHT, L. D. \& J. R. SNOW 1975. The effect of six chemicals for disinfection of largemouth bass eggs. Prog. Fish-Cult. 37: 213-21.

ZAR, J. H. 2010. Biostatistical Analysis. 5th Ed. Pearson Prentice-Hall, Upper Saddle River.

Recibido el 5 de mayo de 2012, aceptado el 22 de abril de 2013. 